\title{
A bioinformatics workflow to detect genes with DNA methylation alterations: a case study of analyzing MeDIP-seq data in cardiac microtissue exposed to epirubicin
}

\author{
Nhan, Nguyen* \\ Department of Toxicogenomics, \\ GROW School for Oncology and \\ Developmental Biology, Maastricht \\ University, Maastricht, The \\ Netherlands \\ nhan.nguyen@maastrichtuniversity.nl
}

\author{
Matthias, Lienhard \\ Max-Planck-Institute for Molecular \\ Genetics, Berlin, Germany \\ lienhard@molgen.mpg.de
}

\author{
Ralf, Herwig \\ Max-Planck-Institute for Molecular \\ Genetics, Berlin, Germany \\ herwig@molgen.mpg.de
}

\author{
Jos, Kleinjans \\ Department of Toxicogenomics, \\ GROW School for Oncology and \\ Developmental Biology, Maastricht \\ University, Maastricht, The \\ Netherlands \\ j.kleinjans@maastrichtuniversity.nl
}

\begin{abstract}
Epigenetic modification such as DNA methylation can be influenced by different exposure conditions. Alterations in DNA methylation status can then lead to changes in gene and protein expressions. Therefore, studying DNA methylation is needed to provide a comprehensive view of cellular mechanisms. One of the costeffective technologies to detect DNA methylation is methylated DNA immunoprecipitation-sequencing (MeDIP-seq). While some tools have been developed to analyze the MeDIP-seq data, they mainly focus on methylated genome regions. Thus, there is still a demand for an analysis process that can detect genes with significant differential methylation. In this study, we developed a workflow built on the QSEA package, a recent launched MeDIP-seq analysis tool in R, to analyze DNA methylation at gene levels. This workflow offers an approach to identify candidate genes with strong methylation alterations. We applied this workflow to analyze MeDIP-seq data from cardiac tissues exposed to epirubicin (EPI), which is an antitumor agent with cardiotoxic adverse effects. Several genes that had strong methylation alteration such as HDAC4, EHMT1, SNHG14, SDHA, IGF1R, ADAP1, and NCOR2, were determined and can be candidates for further EPI-induced cardiotoxicity investigation.
\end{abstract}

${ }^{*}$ Corresponding author

CC.
This work is licensed under a Creative Commons Attribution International
4.0 License.
ICBBB '22, fanuary 07-10, 2022, Tokyo, fapan
(C) 2022 Copyright held by the owner/author(s).
ACM ISBN $978-1-4503-8738-5 / 22 / 01$.
https://doi.org/10.1145/3510427.3510437

\author{
Danyel, Jennen \\ Department of Toxicogenomics, \\ GROW School for Oncology and \\ Developmental Biology, Maastricht \\ University, Maastricht, The \\ Netherlands \\ danyel.jennen@maastrichtuniversity.nl
}

\section{CCS CONCEPTS}

- Applied computing $\rightarrow$ Life and medical sciences; Computational biology; Computational genomics; Life and medical sciences; Computational biology; Recognition of genes and regulatory elements; Life and medical sciences; Bioinformatics.

\section{KEYWORDS}

DNA methylation, epirubicin, MeDIP-seq, toxicogenomics

ACM Reference Format:

Nhan, Nguyen, Matthias, Lienhard, Ralf, Herwig, Jos, Kleinjans, and Danyel, Jennen. 2022. A bioinformatics workflow to detect genes with DNA methylation alterations: a case study of analyzing MeDIP-seq data in cardiac microtissue exposed to epirubicin. In 2022 12th International Conference on Bioscience, Biochemistry and Bioinformatics (ICBBB '22), fanuary 07-10, 2022, Tokyo, Japan. ACM, New York, NY, USA, 7 pages. https://doi.org/10.1145/ 3510427.3510437

\section{INTRODUCTION}

Epigenetic modification can influence the functional state of genome regions without changing the DNA sequence. One of the major epigenetic modifications is DNA methylation, in which a methyl group is added to the fifth carbon position of the cytosine base [1]. DNA methylation is regulated by both methylation and demethylation processes and plays an essential role in different biological activities based on its location in genomic regions. DNA methylation in intergenic regions can repress the expression of potentially harmful genetic elements, while DNA methylation in $\mathrm{CpG}$ islands can diminish transcription factor biding, recruit repressive methyl-binding protein, resulting in gene silencing [2]. In gene regions, DNA methylation at the first exon can lead to gene silencing [2], whereas DNA methylations in other gene regions can also be signals for RNA splicing regulators [3]. Studies 
have determined the relationship between human DNA methylation alterations and external factors such as environmental exposure, nutritional status, and disease $[1,4]$. Furthermore, DNA methylation can become a useful tool to reveal a drug's mode of action and adverse outcome pathway [5]. Thus, DNA methylations, as epigenetic signals, can be allied with gene and protein expression studies in order to have an extensive view of biological mechanisms.

Different approaches can be utilized to detect DNA methylation depending on specific biological problems and financial resources [6]. Among these approaches, methylated DNA immunoprecipitation-sequencing (MeDIP-seq) is a cost-effective technology, which requires low DNA input while providing adequate accuracy, genome coverage, and resolution [7]. MeDIP-seq uses a specific antibody to immunoprecipitate methylated DNA; the obtained fractions are then evaluated by high-throughput sequencing. Thus, MeDIP-seq can estimate the relative enrichment of methylated DNA across the genome [6]. Thereafter, the DNA methylation data need to be processed by mapping the sequencing reads to the reference genome, calculating methylation levels, and identifying differentially methylated regions (DMRs). Different computational methods have been developed to process MeDIPseq data such as Batman [8], MEDIPS [9], MeDUSA [10], MeQA [11], and recently QSEA [12]. Among these methods, the most comprehensive pipelines end at determining and annotating DMRs between samples [13]. Researchers have used MeDIP-seq data analysis methods to address specific research questions, and they usually analyzed DNA methylation at the DMRs level [14, 15]. Only some studies have pursued DNA methylation analysis into gene level by identifying genes that had DMR located in the promoter region [16]. Thus, exploring different ways to analyze DNA methylation at gene levels could elevate the application of DNA methylation analysis.

In this report, we propose a workflow built on the recent developed MeDIP-seq analysis method (QSEA) to address the DNA methylation at the gene levels. The code is written in $\mathrm{R}$ and is available through GitHub for open access. The MeDIP-seq data used in this study was extracted from cardiac microtissues exposed to epirubicin (EPI). While EPI is an important anticancer agent, it can cause short-term heart failure at a very high dose (around $900 \mathrm{mg} / \mathrm{m} 2$ ) and substantial cardiotoxicity at lower doses [17]. By analyzing the methylation status of cardiac tissues exposed to EPI, we identified candidate genes that had strong DNA methylation alteration related to the EPI-induced mechanism.

\section{MATERIALS AND METHODS}

\subsection{Dataset}

The MeDIP-seq data were obtained from the HeCaToS project. In this project, human cardiac microtissues consisting of 4000 iPSCderived human cardiomyocytes from a female Caucasian donor and 1000 cardiac fibroblasts from a male Caucasian donor were exposed to EPI with either therapeutic dose or toxic dose (IC20) based on PBPK modeling for 2 weeks [18]. EPI was dissolved in $0.1 \%$ DMSO before utilization, thus control samples were also exposed to similar DMSO concentrations over time. The microtissues were then collected in triplicates at 2, 8, 24, 72, 168, 240, and 336 hours.
After DNA extraction, the methylated DNA fragments were isolated by anti-5-methylcytosine antibody and then paired-end sequenced (MeDIP-seq) with 50 bp read length [19].

\subsection{Analysis procedure}

MeDIP-seq paired-end reads were aligned to human reference genome hg38 using Burrows-Wheeler Alignment tool (BWA) version 0.7.17 [20] and converted to .bam files using Samtools version 1.10 [21] in Linux environment (Figure 1)..

Thereafter, the aligned MeDIP-seq data were processed in R version 3.6.3 [22] using the QSEA package and human genome build hg38 with default window size (250 bases) excluding sex chromosomes [12]. Copy Number Variations (CNVs) were calculated from input and MeDIP reads based on 1 megabase windows. The $\mathrm{CpG}$ enrichment was estimated using the 'Blind calibration' option. The quality of the mapped MeDIP-seq data was performed using getOffset() and plotEPmatrix() functions. Thereafter, the QSEA package detected DMRs ( $p$-value $<0.01$ ) using generalized linear models (GLMs) with pairwise comparisons between EPI-treated and control samples.

The DMRs were annotated to genomic regions using the "annotar" package [23]. The "annotatr" package provides comprehensive annotations and genomic context of DMRs. The average p-value and $\log 2$ fold change of each gene were calculated based on the average of the p-value and log2 fold change from all DMRs assigned to that gene. Thereafter, interested genes were selected for further analysis based on criteria: (i) top 5\% genes with the high number of DMRs over their gene regions, (ii) top 5\% genes with the high number of DMRs in their promoter region, and (iii) the absolute $\log 2$ fold change $(\log 2 \mathrm{FC})>=0.5$ (Figure 1).

\section{RESULTS}

\subsection{Methylation analysis of all EPI-treated samples compared to control}

There was sufficient methylation enrichment efficiency in the MeDIP-seq data from all samples (Figure 6). In general, DNA methylation profiles differed between EPI-treated and control samples (Figure 2). This indicates that EPI treatment could significantly alter the DNA methylation in cardiac tissues and MeDIP-seq was able to capture these DNA methylation alterations.

There are 183,156 DMRs regions corresponded to 19,825 genes that were detected in the methylation analysis between all EPItreated and control samples. Per gene, the high amount of DMRs, especially in the promoter region, suggests a strong influence of the EPI treatment on the DNA methylation status of that gene compared to control samples. In this case study, 966 genes were in the top $5 \%$ genes that had the highest number of DMR regions per gene (Figure 3A). Thereafter, we selected 47 genes that had the highest number DMRs per promoter region (top 5\% of 966 genes) (Figure 3B). We also enhanced the filtering criteria by only selecting genes that had average absolute $\log 2$ fold change $>=0.5$. Based on all these selection criteria (Figure 1), 35 genes with strong methylated alterations were determined for further analysis. These selected genes were shown in the volcano plot (Figure 4). 

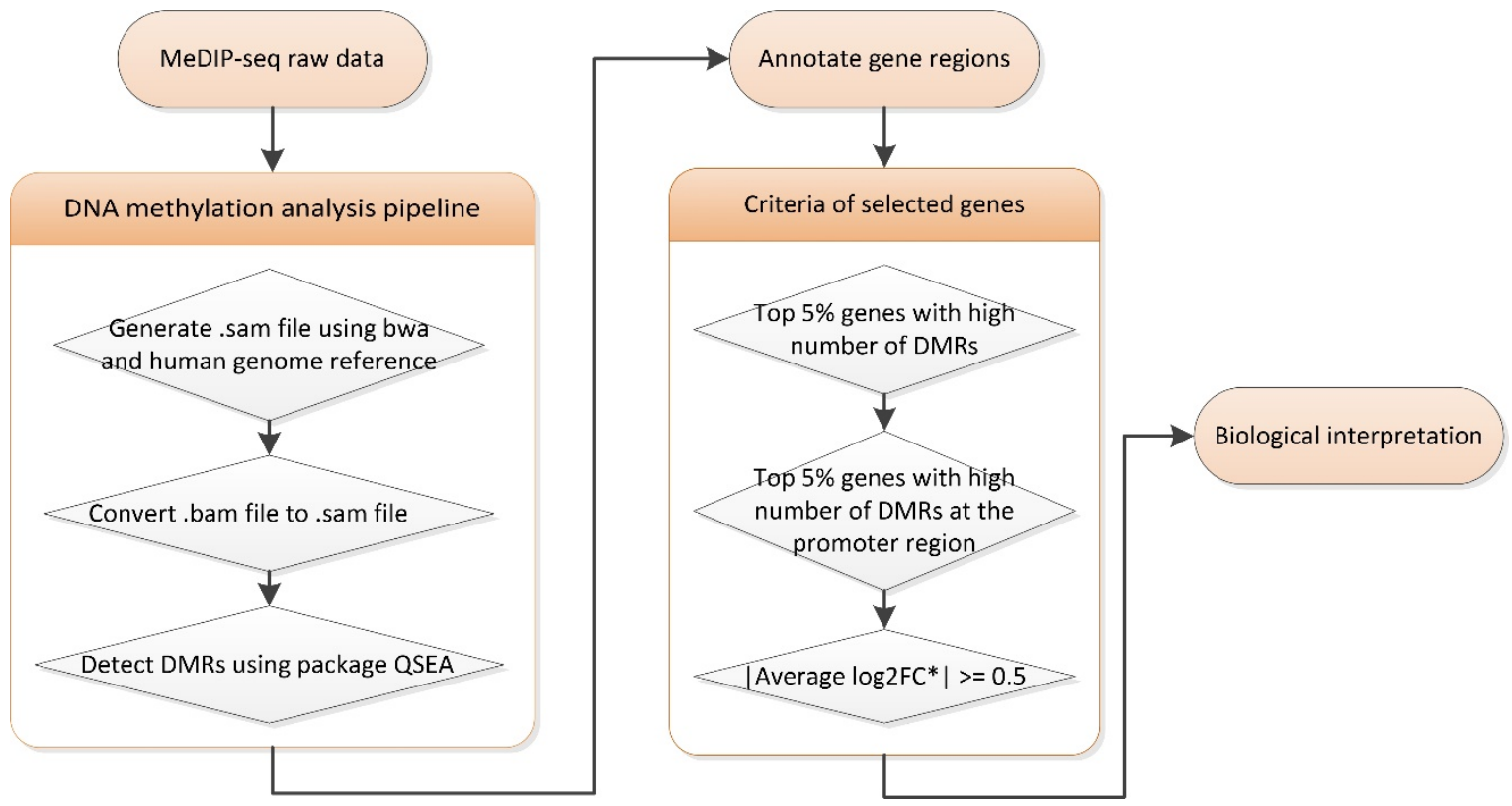

Figure 1: The bioinformatics workflow for MeDIP-seq data analysis. $\left.{ }^{*}\right)$ The average log2FC of each gene was the average log2 FC from all DMRs assigned to that gene.

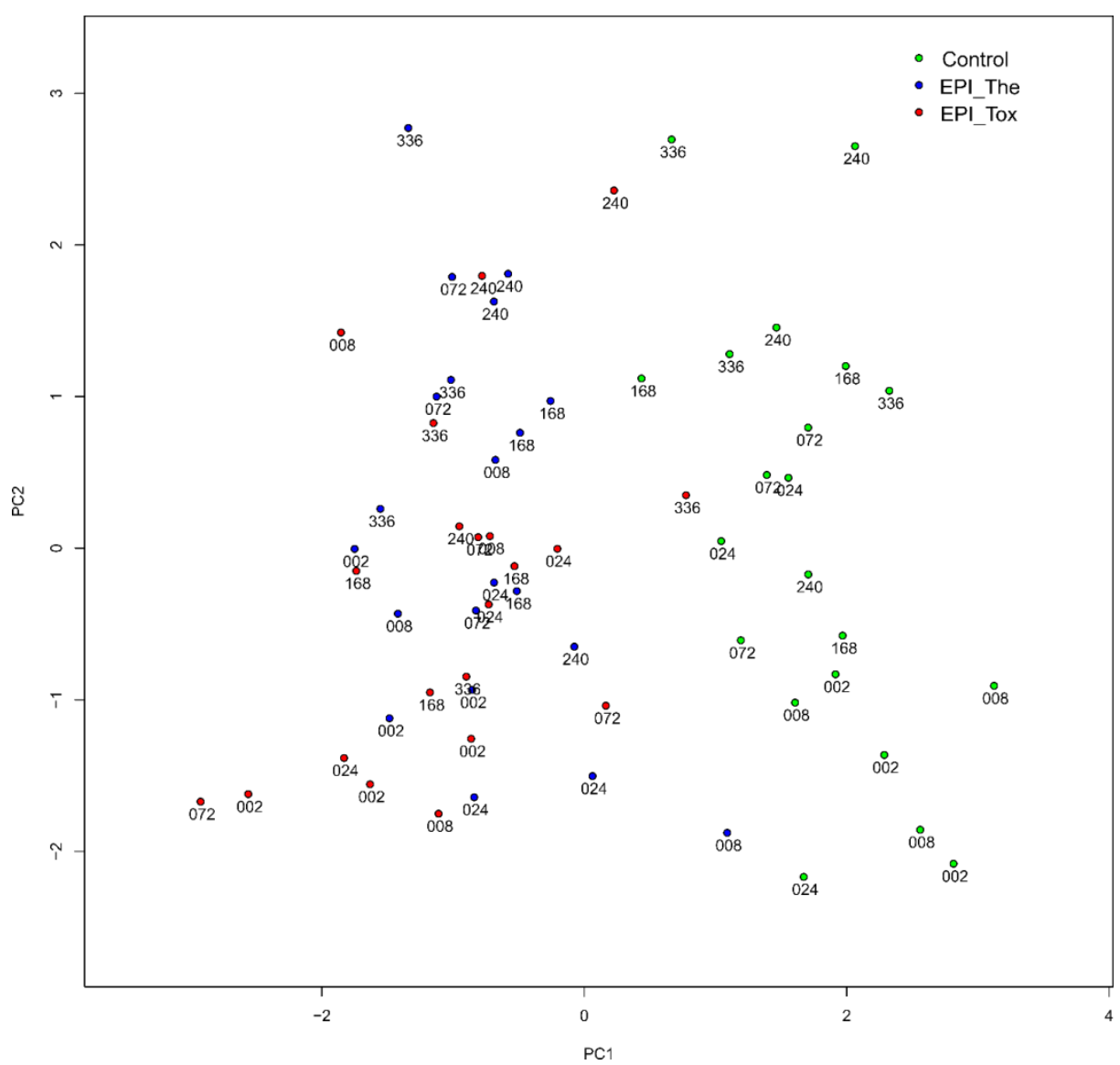

Figure 2: The PCA plots of all EPI-treated and control samples. The numbers are the exposure times in hours. 

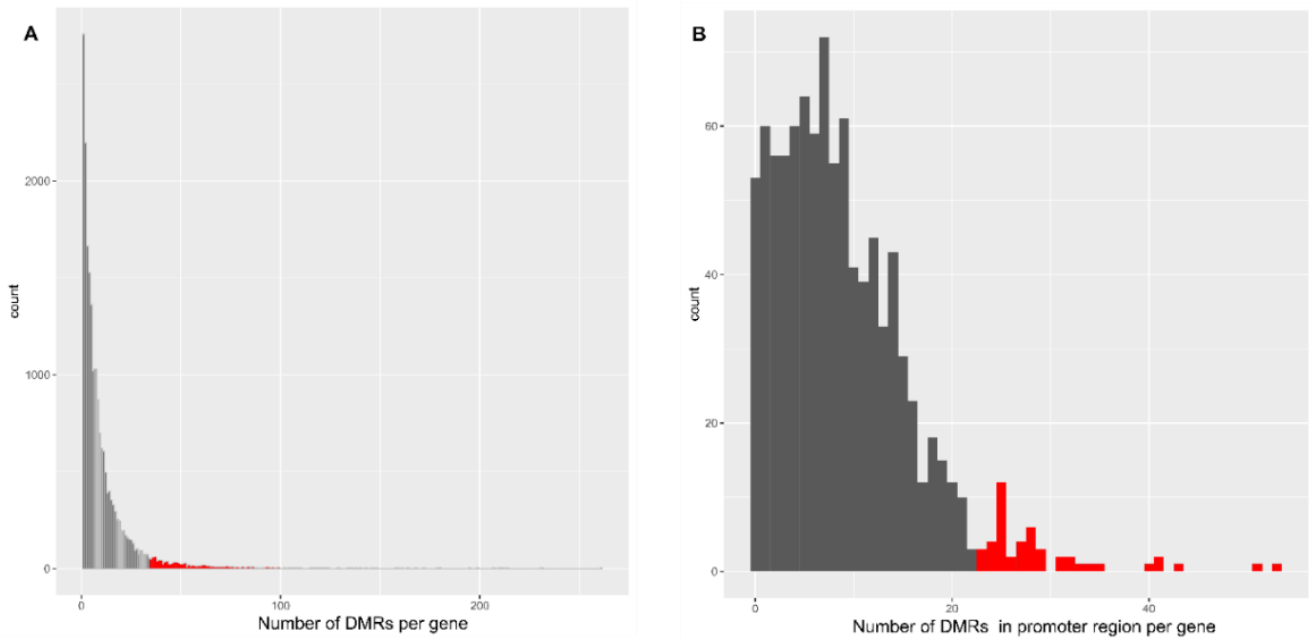

Figure 3: The distribution of genes according to the number of DMRs per gene (A), and the number of DMRs in the promoter region per gene $(B)$ after the differentially methylated analysis between all EPI-treated samples and control samples. The red area indicates selected genes for the next analysis steps (Figure 1).

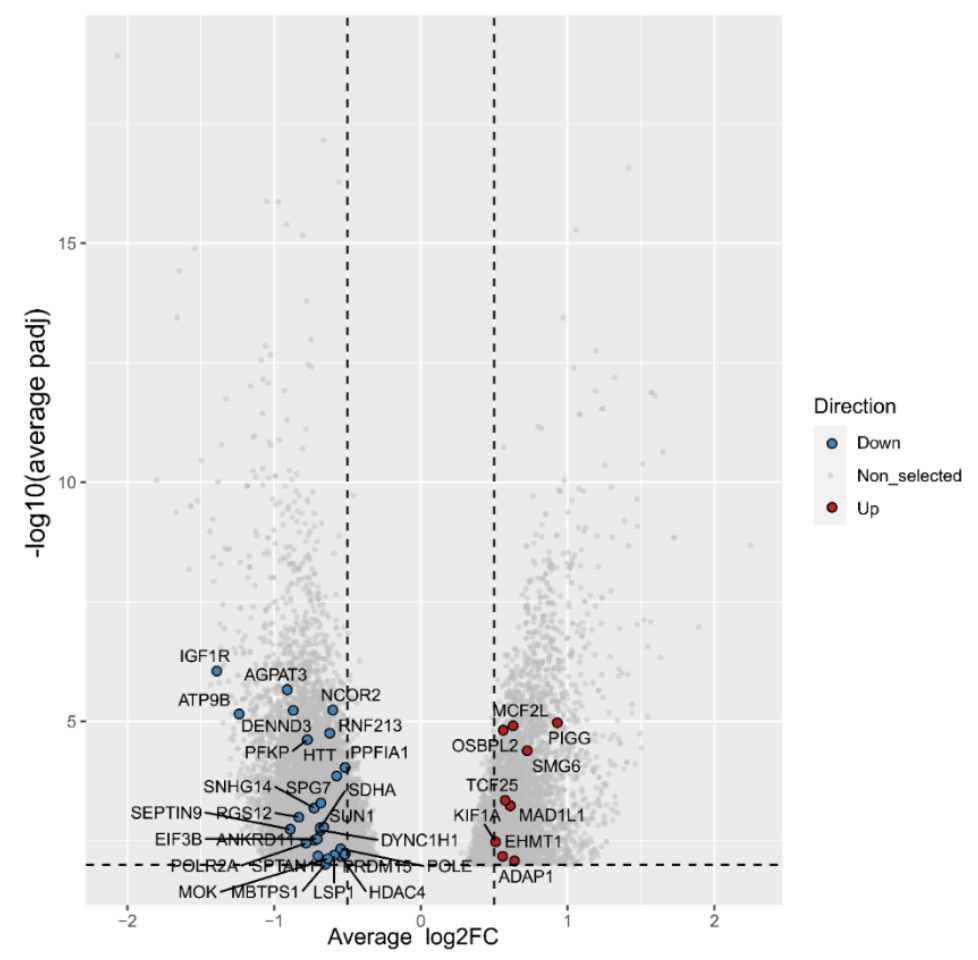

Figure 4: The volcano plot of the differential methylated genes in EPI-treated samples compared to control. Genes that satisfied all the selection criteria (Figure 1) were highlighted in blue and red for hypo-methylated and hyper-methylated status respectively. 

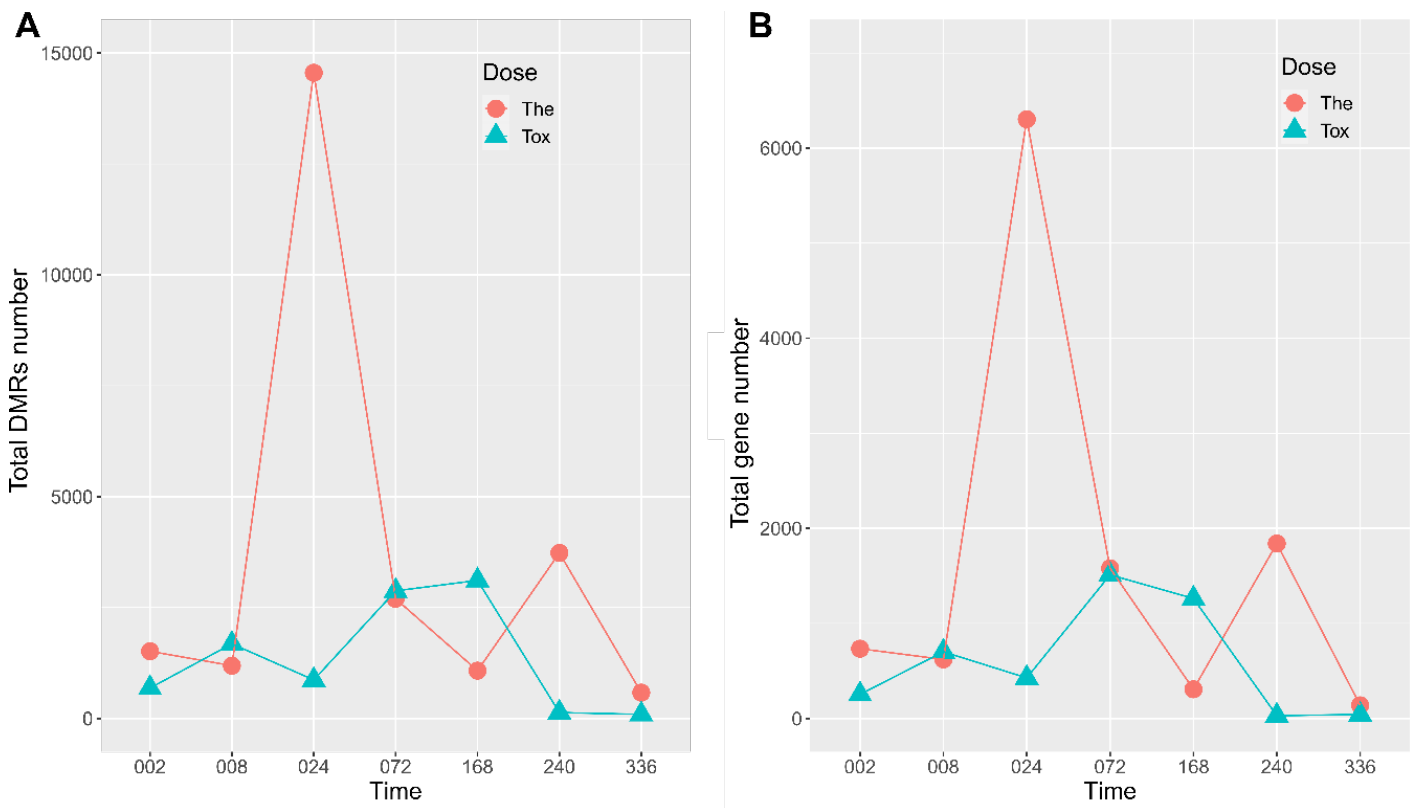

Figure 5: The number of DMRs (A) and corresponding annotated genes (B) after the differentially methylated analysis between EPI-treated and control samples per time exposure and dose.

\subsection{Methylation analysis between EPI-treated and control sample per time exposure and dose}

We applied a similar procedure (Figure 1) to analyze the DMRs and corresponding differential methylated genes between EPI-treated and control samples per time exposure and dose (Figure 5). At some time exposure, the number of DMRs and corresponding genes in samples treated with EPI therapeutic dose was relatively higher than that in samples treated with EPI toxic dose, especially at 24 hours of exposure. Furthermore, the number of DMRs in samples treated with EPI toxic dose undoubtedly reduced from 240 hours of exposure.

\section{DISCUSSION}

Several tools have been established to analyze MeDIP-seq data; however, most of them were published quite a long time ago such as Batman[8] which was launched in 2008, or some tools are currently unavailable such as MeQA [11]. QSEA is a MeDIP analysis $\mathrm{R}$ package that has been recently developed by Lienhard et al. in 2017 to quantify MeDIP-seq data and detect differentially methylated regions between samples [12]. However, there is still a demand for interpreting the differentially methylated status between samples from genome regions toward the gene levels. In this study, we developed a workflow to detect genes with DNA methylation alterations built on the QSEA package. This workflow not only obtain DMRs but it also determine genes that had strong methylation alterations. Thereby, this workflow could elevate the application of DNA methylation analysis.

We utilized this workflow to analyze MeDIP-seq data in cardiac microtissue exposed to epirubicin. Generally, the DNA methylation status in cells can be influenced by disease progression [24] and drug treatment [25]. EPI is a popular chemotherapeutic agent with cardiotoxic adverse effects [17]. Although different studies have investigated the impact of EPI on cellular mechanisms at RNA [26] and protein [27] levels, there is not much research on the epigenetic effect of EPI, especially on DNA methylation level. This study explored the genome-wide DNA methylation alteration under EPI treatment. Candidate genes that changed their methylation status were detected by comparing the DNA methylation between all EPI-treated and control samples (Figure 4). Furthermore, we also compared the DNA methylation status of EPI-treated to control samples per EPI dose and time exposure, It shows that the differences in dose and time exposure can result in different numbers of DMRs and corresponding genes (Figure 5).

The methylation status of some genes changed under EPI treatment (Figure 4) and seem to be associated with EPI cardiotoxic adverse effects. For instance, HDAC4, EHMT1, and SNHG14 are involved in regulatory processes. HDAC4 (Histone Deacetylase 4) was hypomethylated under EPI treatment, and it controls the rapid modification of histone methylation in response to elevated cardiac load [28]. EHMT1 or Euchromatic Histone Lysine Methyltransferase 1 is a histone methyltransferase [29] and was hypermethylated under EPI treatment. The inactivation of this gene relates to cardiac hypertrophy [30]. By contrast, SNHG14 is a non-coding RNA and belongs to a verified regulatory pathway of SNHG14/miR-3225p/miR-384-5p/PCDH17 which can cause repression on hypertrophy in cardiomyocytes [31]. Other hypomethylated genes under EPI treatment encode proteins involved in specific functional processes. For example, SDHA participates in the tricarboxylic acid cycle and mitochondrial respiratory chain; the change of SDHA 
activity can impact mitochondrial acetyl-CoA homeostasis and energy metabolic which contribute to heart failure [32]. IGF1R was hypo-methylated (Figure 4), can enhance the survival of cardiac cells through the regulation of autophagy and apoptosis by induction of the PI3K/Akt pathway [33]. Other protein-coding genes such as ADAP1 and NCOR2 also play roles in cardiac hypertrophy [34] and cardiomyopathy [35, 36]. All these genes had strong DNA methylated alterations in EPI-treated samples compared to control and can be potential targets for EPI-induced cardiotoxicity research.

In conclusion, this study established a DNA methylation analysis workflow for MeDIP-seq data based on the traditional QSEA pipeline in $\mathrm{R}$. This workflow could detect differential methylated status at gene levels and provides an alternative for studying DNA methylation. Furthermore, this study demonstrated initial results about the change of DNA methylation under EPI exposure. Several genes with strong methylation alteration can be candidates for further EPI-induced cardiotoxicity investigation, i.e. HDAC4, EHMT1, SNHG14, SDHA, IGF1R, ADAP1, and NCOR2. DNA methylation can, therefore, serve as a potential approach to understand drug mechanisms as well as discovery biomarkers for drug safety and early drug screening.

\section{HISTORY DATES}

Received October 2021; revised November 2021; accepted December 2021

\section{ACKNOWLEDGMENTS}

We would like to thank Olivia Clayton from InSphero and Roche's staffs, who delivered the samples. This work was supported by the 7th Framework Programme of the European Union (FP7/2007-2013), grant no. 602156 for the HeCaToS project.

\section{REFERENCES}

[1] Martin EM, Fry RC. Environmental Influences on the Epigenome: ExposureAssociated DNA Methylation in Human Populations. 2018;39:309-33.

[2] Moore LD, Le T, Fan G. DNA methylation and its basic function. Neuropsychopharmacology. 2013;38:23-38.

[3] Lev Maor G, Yearim A, Ast G. The alternative role of DNA methylation in splicing regulation. Trends in Genetics. 2015;31:274-80.

[4] Jin Z, Liu Y. DNA methylation in human diseases. Genes \& Diseases. 2018;5:1-8.

[5] Meehan RR, Thomson JP, Lentini A, Nestor CE, Pennings S. DNA methylation as a genomic marker of exposure to chemical and environmental agents. Current Opinion in Chemical Biology. 2018;45:48-56.

[6] Rauluseviciute I, Drabløs F, Rye MB. DNA methylation data by sequencing experimental approaches and recommendations for tools and pipelines for data analysis. Clinical Epigenetics. 2019;11:193.

[7] Taiwo O, Wilson GA, Morris T, Seisenberger S, Reik W, Pearce D, et al. Methylome analysis using MeDIP-seq with low DNA concentrations. Nature Protocols. 2012;7:617-36.

[8] Down TA, Rakyan VK, Turner DJ, Flicek P, Li H, Kulesha E, et al. A Bayesian deconvolution strategy for immunoprecipitation-based DNA methylome analysis. Nat Biotechnol. 2008;26:779-85.

[9] Chavez L, Jozefczuk J, Grimm C, Dietrich J, Timmermann B, Lehrach H, et al. Computational analysis of genome-wide DNA methylation during the differentiation of human embryonic stem cells along the endodermal lineage. Genome Res. 2010;20:1441-50.

[10] Wilson GA, Dhami P, Feber A, Cortázar D, Suzuki Y, Schulz R, et al. Resources for methylome analysis suitable for gene knockout studies of potential epigenome modifiers. GigaScience. 2012;1.

[11] Huang J, Renault V, Sengenès J, Touleimat N, Michel S, Lathrop M, et al. MeQA: a pipeline for MeDIP-seq data quality assessment and analysis. Bioinformatics (Oxford, England). 2012;28:587-8

[12] Lienhard M, Grasse S, Rolff J, Frese S, Schirmer U, Becker M, et al. QSEA-modelling of genome-wide DNA methylation from sequencing enrichment experiments.
Nucleic Acids Res. 2017;45:e44-e

[13] Wilson GA, Beck SJNGS-A, Applications, Challenges. Computational analysis and integration of MeDIP-seq methylome data. 2016.

[14] Beck D, Sadler-Riggleman I, Skinner MK. Generational comparisons (F1 versus F3) of vinclozolin induced epigenetic transgenerational inheritance of sperm differential DNA methylation regions (epimutations) using MeDIP-Seq. Environmental Epigenetics. 2017;3.

[15] Dworkin M, Xie S, Saha M, Thimmapuram J, Kalavacharla V. Analyses of methylomes of upland and lowland switchgrass (Panicum virgatum) ecotypes using MeDIP-seq and BS-seq. BMC Genomics. 2017;18:851

[16] Shi Y, Gong W, Gong X, Wang P, Zhao X. Genome-wide DNA methylation analysis of breast cancer MCF-7 / Taxol cells with MeDIP-Seq. PLOS ONE. 2020;15:e241515.

[17] Mahmood SS, Patel RB, Butler J, Vaduganathan M. Epirubicin and long-term heart failure risk in breast cancer survivors. Eur J Heart Fail. 2018;20:1454-6.

[18] Kuepfer L, Clayton O, Thiel C, Cordes H, Nudischer R, Blank LM, et al. A modelbased assay design to reproduce in vivo patterns of acute drug-induced toxicity. Archives of Toxicology. 2018;92:553-5.

[19] Selevsek N, Caiment F, Nudischer R, Gmuender H, Agarkova I, Atkinson FL, et al. Network integration and modelling of dynamic drug responses at multi-omics levels. Commun Biol. 2020;3:573-.

[20] Li H, Durbin R. Fast and accurate short read alignment with Burrows-Wheeler transform. Bioinformatics (Oxford, England). 2009;25:1754-60.

[21] Danecek P, Bonfield JK, Liddle J, Marshall J, Ohan V, Pollard MO, et al. Twelve years of SAMtools and BCFtools. Gigascience. 2021;10.

[22] R Core Team. R: A Language and Environment for Statistical Computing. Vienna, Austria: R Foundation for Statistical Computing; 2020.

[23] Cavalcante RG, Sartor MA. annotatr: genomic regions in context. Bioinformatics (Oxford, England). 2017;33:2381-3.

[24] Dreval K, Tryndyak V, de Conti A, Beland FA, Pogribny IP. Gene Expression and DNA Methylation Alterations During Non-alcoholic Steatohepatitis-Associated Liver Carcinogenesis. 2019;10.

[25] Zhang K, Li C, Liu J, Tang X, Li Z. DNA methylation alterations as therapeutic prospects in thyroid cancer. Journal of Endocrinological Investigation. 2019;42:363-70.

[26] Du T, Shi Y, Xu S, Wan X, Sun H, Liu B. Long Non-Coding RNAs in Drug Resistance of Breast Cancer. Onco Targets Ther. 2020;13:7075-87.

[27] Nguyen N, Souza T, Verheijen MCT, Gmuender H, Selevsek N, Schlapbach R, et al. Translational Proteomics Analysis of Anthracycline-Induced Cardiotoxicity From Cardiac Microtissues to Human Heart Biopsies. Front Genet. 2021;12:695625-.

[28] Hohl M, Wagner M, Reil J-C, Müller S-A, Tauchnitz M, Zimmer AM, et al. HDAC4 controls histone methylation in response to elevated cardiac load. The Journal of Clinical Investigation. 2013;123:1359-70.

[29] Stelzer G, Rosen N, Plaschkes I, Zimmerman S, Twik M, Fishilevich S, et al. The GeneCards Suite: From Gene Data Mining to Disease Genome Sequence Analyses. 2016;54:1.30.1-1..3.

[30] Thienpont B, Aronsen JM, Robinson EL, Okkenhaug H, Loche E, Ferrini A, et al. The H3K9 dimethyltransferases EHMT1/2 protect against pathological cardiac hypertrophy. The Journal of Clinical Investigation. 2017;127:335-48.

[31] Long Y, Wang L, Li Z. SP1-induced SNHG14 aggravates hypertrophic response in in vitro model of cardiac hypertrophy via up-regulation of PCDH17. 2020;24:711526.

[32] Horton JL, Martin OJ, Lai L, Riley NM, Richards AL, Vega RB, et al. Mitochondrial protein hyperacetylation in the failing heart. JCI Insight. 2016;2:e84897.

[33] Kwon MJ, Nam TJ. A polysaccharide of the marine alga Capsosiphon fulvescens induces apoptosis in AGS gastric cancer cells via an IGF-IR-mediated PI3K/Akt pathway. Cell biology international. 2007;31:768-75.

[34] Giguère H, Dumont A-A, Berthiaume J, Oliveira V, Laberge G, Auger-Messier M. ADAP1 limits neonatal cardiomyocyte hypertrophy by reducing integrin cell surface expression. Scientific Reports. 2018;8:13605.

[35] Haidar MN, Islam MB, Chowdhury UN, Rahman MR, Huq F, Quinn JMW, et al. Network-based computational approach to identify genetic links between cardiomyopathy and its risk factors. IET Syst Biol. 2020;14:75-84

[36] Cividini F, Scott BT, Suarez J, Casteel DE, Heinz S, Dai A, et al. Ncor2/PPAR $\alpha$ Dependent Upregulation of MCUb in the Type 2 Diabetic Heart Impacts Cardiac Metabolic Flexibility and Function. 2021;70:665-79.

\section{A APPENDICES}

\section{A DATA \& CODE}

The MeDIP-seq data are from the HeCaToS project and are deposited in BioStudies (http://www.ebi.ac.uk/biostudies) under accession numbers S-HECA433 and S-HECA434. The R code is available on Github (https://github.com/NhanNguyen000/MeDIP.. 


\section{B SUPPLEMENTARY FIGURES}

\section{CpG Enrichment Analysis}
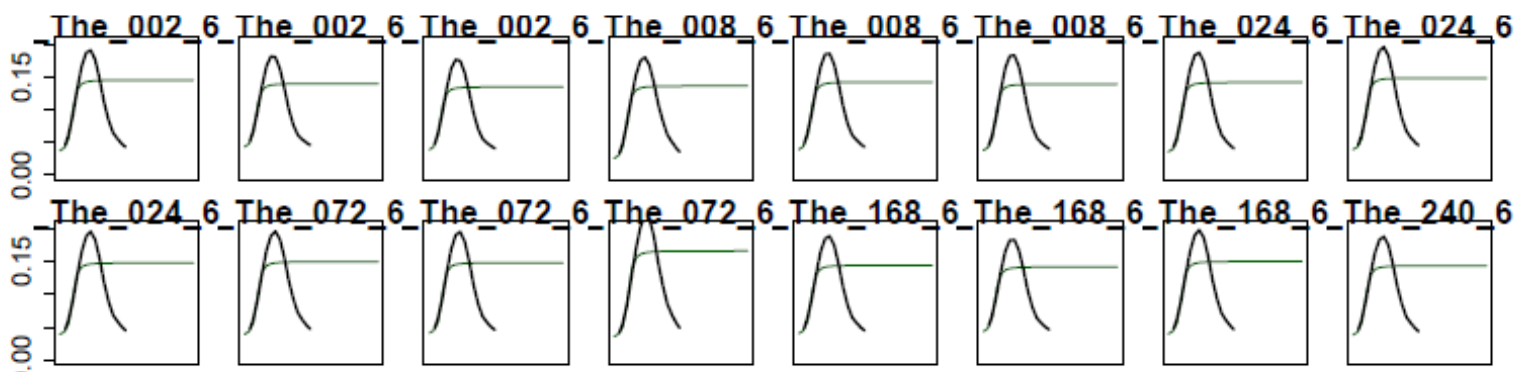

$\circ$
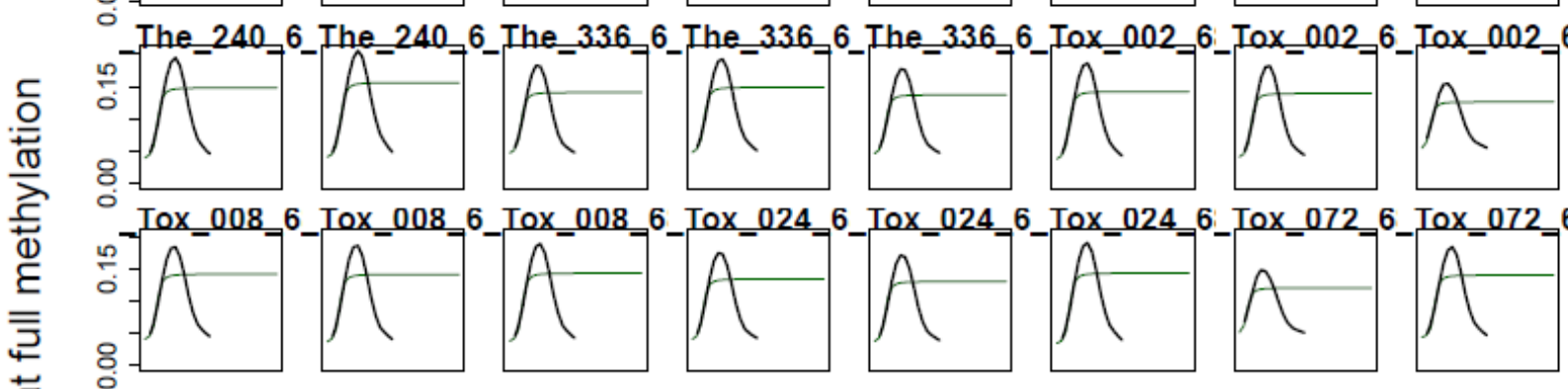

Tox_008_6_Tox_008_6_Tox_008_6_Tox_024_6_Tox_024_6_Tox_024_6_Tox_072_6_Tox_072_6

8
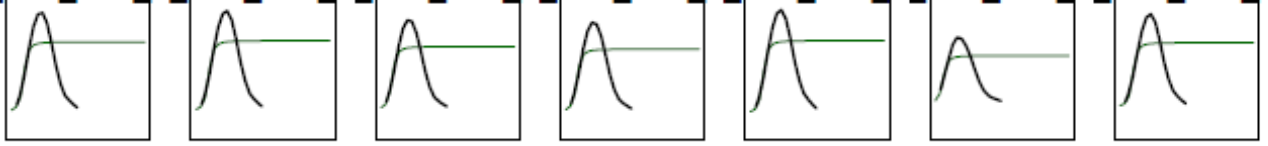

Tox 072
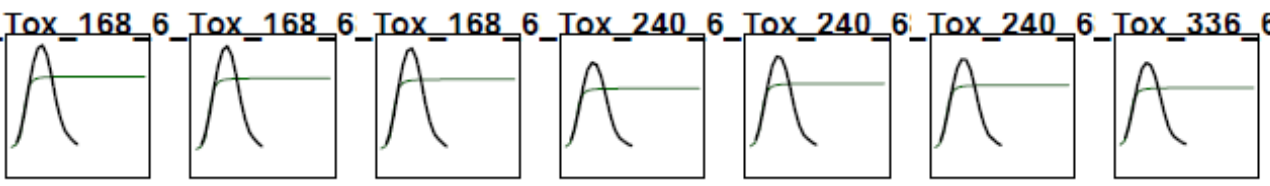

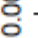

Tox_336
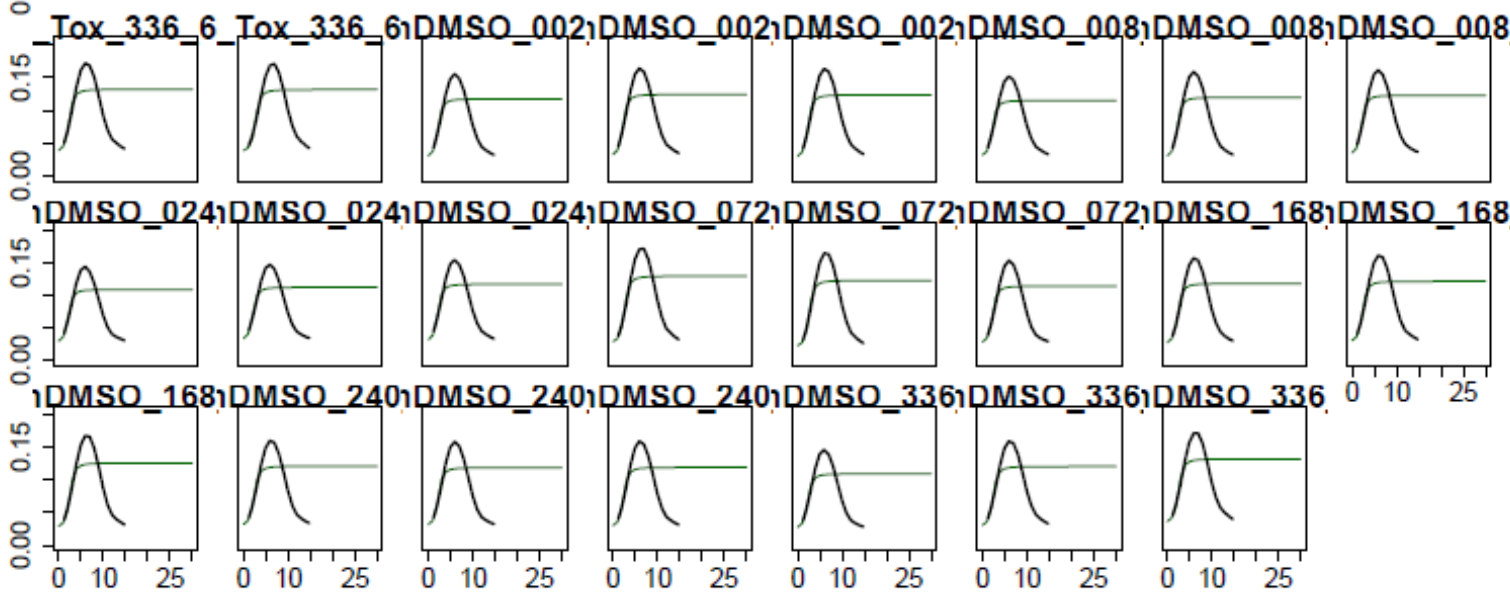

CpG density [\#/fragment]

Figure 6: The sample-wise CpG density-dependent enrichment profiles. The average enrichment profile of samples is depicted in black, and the fitted sigmoidal function is in green. Samples with flat profiles might indicate low enrichment efficiency or poor agreement with the calibration data. In here, all samples show sufficient enrichment profiles. 